International Journal of Engineering \& Technology, $7(2.13)(2018)$ 306-308
International Journal of Engineering \& Technology
Website: $w$ ww.sciencepubco.com/index.php/IJET
Research Paper

\title{
Unit regular inverse monoids and Cliford monoids
}

\author{
Sreeja V. K. * \\ Department of Mathematics, Amrita Vishwa Vidyapeetham, Amritapuri, India \\ *Corresponding author E-mail: sreeja@amritapuri.amrita.edu
}

\begin{abstract}
Let $S$ be a unit regular semigroup with group of units $G=G(S)$ and semilattice of idempotents $E=E(S)$. Then for every $x \in S$ there is a $u \in G$ such that $x=x u x$. Then both $x u$ and $u x$ are idempotents and we can write $x=u^{-1} u x$ or $x=x u u^{-1}$. Thus every element of a unit regular inverse monoid is a product of a group element and an idempotent. It is evident that every $L$-class and every $R$-class contains exactly one idempotent where $L$ and $R$ are two of Greens relations. Since inverse monoids are $R$ unipotent, every element of a unit regular inverse monoid can be written as $s=e u$ where the idempotent part $e$ is unique and $u$ is a unit. A completely regular semigroup is a semigroup in which every element is in some subgroup of the semigroup. A Clifford semigroup is a completely regular inverse semigroup. Characterization of unit regular inverse monoids in terms of the group of units and the semilattice of idempotents is a problem often attempted and in this direction we have studied the structure of unit regular inverse monoids and Clifford monoids.
\end{abstract}

Keywords: Inverse Monoids; Unit Regular Monoids; Clifford Monoids; Green's Relation.

\section{Preliminaries}

Throughout the current research let $\mathrm{E}(\mathrm{S})$ denote the semilattice of idempotents and $\mathrm{G}(\mathrm{S})$ note the collection of units of the regular monoid S. In this paper, two problems of linear and nonlinear inverse heat conduction and also a nonlinear inverse parabolic problem are investigated in the third chapter. The first problem related to the estimate of heat flux in the boundary is a reverse heat conduction problem with the heat source. Given the above additional condition, an integral union is proved in terms of the false heat flux. Then, using the finite difference method, the unknown flux is estimated in terms of the first-order Chebyshev orthogonal functions. The second problem is the simultaneous estimation of two unknowns in boundary conditions of a linear heat conduction problem. Given the above conditions, the problem is initially proved to be inverse problem and using the CrankNicholson method and the method of approximation of the function, the unknown functions are estimated and the results are compared by providing an example. In the last problem, the determination of the temperature-dependent penetration coefficient in a nonlinear parabolic problem is investigated. Given the additional condition of the problem, the existence of the problem is proved. In the following, the coefficient of infiltration is estimated using the Tihonov ordering method. The regulator parameter is determined in terms of the initial input error and is estimated with the approximation of the Euler-Lagrange equation dependent on the aid of the finite difference function of the unknown function.

Proposition 1.3. ([2]).

Proof: First we observe that for $\mathrm{e} \in \mathrm{E}, \mathrm{e} \rightarrow \mathrm{g} * \mathrm{e}=\mathrm{geg}^{-1}$ is a biorder isomorphism of $\mathrm{E}$ for each $\mathrm{g} \in \mathrm{G}$. Further if $\mathrm{g}_{1}, \mathrm{~g}_{2} \in \mathrm{G}$ and $\mathrm{e} \in \mathrm{E}$, then

$\left(g_{1} g_{2}\right) * e=g_{1} g_{2} e\left(g_{1} g_{2}\right)^{-1}$

$=\mathrm{g}_{1} \mathrm{~g}_{2} \mathrm{eg}_{2}{ }^{-1} \mathrm{~g}_{1}{ }^{-1}$

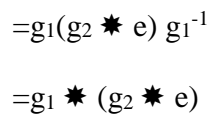

Also,

$1 * \mathrm{e}=\mathrm{e}$.

Let $\mathrm{R}$ and $\mathrm{L}$ be two of Green's relations. Then we have the following proposition.

Proposition 1.4. ([1]).

Let $S$ be an inverse monoid with $\mathrm{G}=\mathrm{G}(\mathrm{S})$ and $\mathrm{E}=\mathrm{E}(\mathrm{S})$. Then the following conditions are equivalent on $\mathrm{S}$. (i) $\mathrm{S}$ is unit regular (ii) $L_{e}=G e$ for every $e \in E$. (iii) $R_{e}=e G$ for every $e \in E$, where $L_{e}$ is the L-class containing e and $R_{e}$ is the R-class containing e.

Proposition 1.5. ([2]).

The homomorphic images of unit regular monoids are unit regular. It is well known that the set of idempotents $E(S)$ of an inverse semigroup $S$ is a semilattice. Further the relations $L \mid E(S)$ and $R \mid E(S)$ are trivial, where $\mathrm{L}$ and $\mathrm{R}$ are Greens relations.

In this article, we will review the linear and invertible problems that appear in many practical application Representation of unit regular inverse monoids and Clifford monoids The instability of a particular type of these problems (the Fredholm type-1 integral equation) and its relation to the kernel of the equation are also examined.

We need some sort of regulation to get the right answers for these issues. In the end, using the answer system for a singledimensional boundary value problem.

Proposition 2.1. [4]

Proof: Still, $(\mathrm{e}, \mathrm{u}) \sim(\mathrm{e}, \mathrm{u})$, till eu $=$ eu. So $\sim$ is reflexive. Still propose $(\mathrm{e}, \mathrm{u}) \sim(\mathrm{f}, \mathrm{v})$. Hence $\mathrm{e}=\mathrm{f}$ and $\mathrm{uv}^{-1} \in \mathrm{G}(\mathrm{e})$. Till $\mathrm{f}=\mathrm{e}$ and $\mathrm{vu}^{-1}$ $\in \mathrm{G}$ (e) we get (f, v) (e, u). So $\sim$ is symmetric. Still propose (e, $\mathrm{u}) \sim(\mathrm{f}, \mathrm{v}) \sim(\mathrm{g}, \mathrm{w})$. So $\mathrm{e}=\mathrm{f}$ and $\mathrm{f}=\mathrm{g}$. In addition $\mathrm{uv}^{-1} \mathrm{v} \mathrm{w}^{-1} \in \mathrm{G}$ (e). So $(e, u) \sim(e, w)$. So $\sim$ is transitive. 
Remark: We denote the equivalence class of $(e, u)$ by $[e, u]$ and the quotient $(E \times G) / \sim$ by T.

If $\mathrm{S}$ is a unit regular inverse monoid, on $\mathrm{E}(\mathrm{S})$ we define the relations $\omega^{r}$ and $\omega^{\prime}$ as follows. $\omega^{r}=\{(e, f): f e=e\}, \omega^{l}=\{(e, f): e f=e\}$.

Also it is evident that $R \mid E(S)=\omega^{r} \cap\left(w^{r}\right)^{-1}$ and

$L \mid E(S)=\omega^{\prime} \cap\left(w^{\prime}\right)^{-1}$ are trivial relations where $\mathrm{L}$ and $\mathrm{R}$ are Green's relations on $\mathrm{S}$.

Proposition 2.2

Let $S$ be a unit regular inverse monoid. Suppose that product in $\mathrm{T}$ is defined as $[e, u][f, v]=[e(u * f)$, uv].Then $T$ is a inverse unit regular monoid isomorphic to $\mathrm{S}$ with set of idempotents given by

$E(T)=\{[e, 1]: e \in E\}$

And collection of units given by

$\mathrm{G}(\mathrm{T})=\{[1, \mathrm{u}]: \mathrm{u} \in \mathrm{G}\}$

Proof: Now let $\mathrm{x} \in \mathrm{S}$. Then $\mathrm{x}=\mathrm{eu}$ where $\mathrm{e} \in \mathrm{E}$ and $\mathrm{u} \in \mathrm{G}$, by proposition 1.1. Now define $\varphi: S \rightarrow T$ as $(x) \varphi=(e u) \varphi=[e, u]$. First we show that $\varphi$ is well defined. Since $\mathrm{S}$ is an inverse unit regular monoid we can express $x=e u=e v$. For if $\mathrm{x}=\mathrm{eu}=\mathrm{fv}$ where $\mathrm{e}, \mathrm{f} \in \mathrm{E}$ and $\mathrm{u}, \mathrm{v} \in \mathrm{G}$, then $e u v^{-1}=f$. Hence $e R f$. So $e=f$, since $\mathrm{S}$ is an inverse unit regular monoid. Since eu $=\mathrm{ev}$, and $u v^{-1} \in G(e)$ we get $[e, u]=[e, v]$. Now let $x \varphi=y \varphi$ where $x=e u$ and $y=f v$. Then $[e$, $\mathrm{u}]=[\mathrm{f}, \mathrm{v}]$. Hence $\mathrm{e}=\mathrm{f}$ and $u v^{-1} \in G(e)$.

In this thesis, the inverse problem associated with the parabolic equation is studied. The mathematical model of many issues in the fields of applied science leads to this form. Based on the optimal control process, the existence and minimum necessary condition for the control function is proved. Since the problem of optimal control is non-uniform, in general, one should not expect a unique solution, but by applying the special conditions in this thesis, we prove that the answer is locally unique $[5,6]$.

So eu $=$ ev. That is, $\mathrm{x}=\mathrm{y}$. Hence $\varphi$ is one one. Also (xy) $\varphi=$ (eufv) $\varphi$ where $x=e u$ and $y=f v$. Now eufv $=e^{-u f u^{-1}} u v=e$ ufu ${ }^{1} u v$. Hence (xy) $\varphi=\left(e^{e u f u^{-1}} u v\right) \varphi=\left[e^{-u f u^{-1}}, u v\right]$. Also x $\varphi$ y $\varphi=[e, u]$ $[f, v]=\left[e u f u^{-1}, u v\right]$. Therefore $(x y) \varphi=(x \varphi)(y \varphi)$. Evidently $\varphi$ is onto. So [e, 1] [f, 1] = [e (1*f), 1] = [ef, 1]. Also [f, 1] [e, 1] $=[\mathrm{f}$ $(1 * \mathrm{e}), 1]=[\mathrm{fe}, 1]$. So $[\mathrm{e}, 1][\mathrm{f}, 1]=[\mathrm{f}, 1][\mathrm{e}, 1]$. Hence the idempotents commute each other. So $\mathrm{T}$ is a unit regular inverse monoid.

Theorem 2.3: In case the unit regular inverse monoid is also a Clifford semigroup that is an inverse semigroup in which the idempotents are central we get further simplifications. In the case of E-centralising group $G$ the group action $u * e=e$ for all $u \in G$ and $e \in E$. In that case $G(e)$ is a normal subgroup of $G$.

In applied mathematics, in particular, for determining the approximate solution of the integral, ordinary and partial differential equations, we face problems which, although theoretically, have the same unique solution, but in practice, with their discretization, they have different numerical solutions to the problem. Coming. In such cases, in some way, from the approximate answers, we must choose the answer that is closer to the real answer. After disrupting these types of problems, almost all of them lead to the solution of a linear equation system. The matrix of the coefficients of these devices is oblique and the right vector is disturbed. The deficiency of the coefficient matrix makes it possible to apply marginal errors in the right vector, which makes it extremely difficult to answer the problem.

Proposition 2.4

Let $\mathrm{S}$ be a unit regular inverse monoid. with $\mathrm{E}$ centralising group of units. Also let $\mathrm{E}$ be the set of idempotents and $\mathrm{G}$ be the group of units of an inverse unit regular monoid S Suppose that product in $\mathrm{T}=(E \times G) / \sim$ is defined as $[\mathrm{e}, \mathrm{u}][\mathrm{f}, \mathrm{v}]=[\mathrm{ef}, \mathrm{uv}]$. Then $\mathrm{T}$ is a inverse unit regular monoid isomorphic to $\mathrm{S}$ with set of idempotents given by

$E(T)=\{[e, 1]: e \in E\}$

And group of units given by

$\mathrm{G}(\mathrm{T})=\{[1, \mathrm{u}]: \mathrm{u} \in \mathrm{G}\}$

With E-centralising group of units. That is $\mathrm{T}$ is a unit regular Clifford semigroup.

Proof: By Proposition 2.2, $\mathrm{T}$ is a unit regular inverse monoid. Evidently $[\mathrm{e}, 1][1, \mathrm{u}]=[1, \mathrm{u}][\mathrm{e}, 1]$.

Let $\mathrm{G}(\mathrm{e})=\mathrm{G}$ for all $e \neq 1$ and $G(1)=\{1\}$ in the above Theorem 2.3.

Then we get a unit regular Clifford semigroup. With respect to the notations of the Theorem 2.3 we have the following corollary.

Corollary 2.5

Let $\mathrm{S}$ be a unit regular Clifford semigroup with $\mathrm{G}(\mathrm{e})=\mathrm{G}$ for all $e \neq 1$ and $G(1)=\{1\}$. Then $\mathrm{S} \approx T \approx G \cup(E \backslash\{1\})$

Proof: In Theorem 2.3 we have $\mathrm{G}(\mathrm{e})=\mathrm{G}$ for all $e \neq 1$ and $G(1)=\{1\}$.Then for $e \in E$ and $u, v \in G,(e, u) \sim(f, v)$ if $\mathrm{e}=\mathrm{f}$ and $\mathrm{uv}^{-1} \in \mathrm{G}(\mathrm{e})$. So if $e \neq 1,(\mathrm{e}, \mathrm{u}) \sim(\mathrm{e}, \mathrm{v})$ for all $\mathrm{u}$ and $\mathrm{v}$ and $(1, \mathrm{u}) \sim$ $(1, \mathrm{v})$ only for $\mathrm{u}=\mathrm{v}$. So $e \times G$ is a single equivalence class for $e \neq 1$ which may be identified with e. Also $[1, \mathrm{u}]=\{(1, \mathrm{u})\}$ and this may be identified with $\mathrm{u}$. Thus $(E \times G) / \sim$ can be identified with as $G \cup(E \backslash\{1\})$.

With respect to the notations of the Proposition 1.7 we have the following remark.

Remark 2.6: Every unit regular Clifford semigroup give rise to a collection of normal subgroups $\{G(e): e \in E(S)\}$ of the group of units of $G$. The partial order on the set $E$ of idempotents in a semigroup $S$ is defined as follows. For any e,f in $E(S), e \leq f$ if and only if $e=e f=$ fe. Hence $G(f) \subseteq G(e)$. Since $G(f)$ and $G(e)$ are normal subgroups of $G$ and $G(f) \subseteq G(e)$ the map $\varphi(e, f)$ : $G / G(f) \rightarrow G / G(e)$ defined by $x G(f) \rightarrow x G(e)$ is a surjective homomorphism of groups. We call this homomorphism "coset absorption".

A Clifford semigroup is a strong semilattice union of groups and is described in terms of the semilattice, the groups and connecting group homomorphisms as follows. Let $\mathrm{E}$ be the semilattice and $\left\{\mathrm{N}_{\mathrm{e}} \mathrm{e} \in \mathrm{E}\right\}$ be the groups. Connecting homomorphisms are $\varphi(\mathrm{e}, \mathrm{f})$ : $\mathrm{N}_{\mathrm{f}} \rightarrow \mathrm{N}_{\mathrm{e}}$ whenever $\mathrm{e} \leq \mathrm{f}$. Further the product in the union $\cup_{\mathrm{e} \in \mathrm{E}} \mathrm{N}_{\mathrm{e}}$ is defined for $\mathrm{x} \in \mathrm{N}_{\mathrm{e}}$ and $\mathrm{y} \in \mathrm{N}_{\mathrm{f}}$ by $\mathrm{xy}=\mathrm{x} \varphi(\mathrm{e}$, ef). $\mathrm{y} \varphi$ (f, ef) where the right hand side is is a product in $\mathrm{N}_{\mathrm{ef}}$. We denote the Clifford semigroup obtained in this way by $\left\langle E, N_{e}, \varphi\right\rangle$. In view of the above discussion we have the following characterization for unit regular Clifford semigroups.

With respect to the above notations of the Remark 2.6 we have the following theorem.

Theorem 2.7: Let $S$ be a unit regular Clifford semigroup. Then $U$ $=\left\langle E, N_{e}, \varphi\right\rangle$. is a unit regular Clifford semigroup, where $E=$ $E(S), G=G(S), N_{e}=G / G(e)$ and $\varphi(e, f): G / G(f) \rightarrow G / G(e)$.

In the theory of quantum fields, there are many obvious divergences. Of course, the physical quantities are all finite, so divergences only appear during computational stages. So we need to regulate divergences by different regulators. Two new regulators we introduce the theory of quantum fields that is related to spacetime with an extra dimensional compression on a circle.

\section{Acknowledgement}

I would like to thank Dr. A.R Rajan for his valuable suggestions. 


\section{References}

[1] Chen S.Y. and S.C. Hsieh, Factorizable inverse semigroups, Semi-

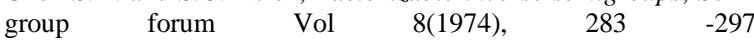
https://doi.org/10.1007/BF02194773.

[2] Hickey J.B and M.V. Lawson, Unit regular monoids, University of Glasgow, Department of Mathematics.

[3] Nambooripad K.S.S., The natural partial order on a regular semigroup, Proc. Edinburgh Math. Soc (1980), 249-260. https://doi.org/10.1017/S0013091500003801.

[4] A.R. Rajan and V.K. Sreeja, Construction of a R-strongly unit regular Monoid from a regular Biordered set and a group, AsianEur. J. Ma.

[5] Mahdi Fakoor, Amirreza Kosari, Mohsen Jafarzadeh, Revision on fuzzy artificial potential field for humanoid robot path planning in unknown environment, International Journal of Advanced Mechatronic Systems, Volume 6, Issue 4, page 174-183, 2015. https://doi.org/10.1504/IJAMECHS.2015.072707.

[6] Fakoor, M., Kosari, A., Jafarzadeh, M. Journal of Applied Research and Technology, Journal of Applied Research and Technology, Volume 14, Issue 5, 2016, 300-310, 2016. 